\title{
Combining FISH with localisation microscopy: Super-resolution imaging of nuclear genome nanostructures
}

\author{
Yanina Weiland • Paul Lemmer • \\ Christoph Cremer
}

Published online: 29 December 2010

(C) Springer Science+Business Media B.V. 2010

\begin{abstract}
The optical resolution of conventional far field fluorescence light microscopy is restricted to about $200 \mathrm{~nm}$ laterally and $600 \mathrm{~nm}$ axially and has been thought for many decades to be an insurmountable barrier for the quantitative spatial analysis of cellular and hence also nuclear constituents. Novel approaches in light microscopy have now overcome this barrier. Here, we report on a special method of localisation microscopy, spectral precision distance/ position determination microscopy and its combination with fluorescence in situ hybridization to analyse the spatial distribution of specific DNA sequences in human cell nuclei at the macromolecular optical resolution level. As an example, repetitive DNA
\end{abstract}

Yanina Weiland and Paul Lemmer contributed equally.

Y. Weiland $\cdot$ P. Lemmer $\cdot$ C. Cremer

Kirchhoff Institute for Physics, University of Heidelberg, Im Neuenheimer Feld 227,

69120 Heidelberg, Germany

C. Cremer

Biophysics of Genome Structure, Institute for Pharmacy and Molecular Biotechnology, Im Neuenheimer Feld 364, University of Heidelberg,

69120 Heidelberg, Germany

C. Cremer $(\bowtie)$

Institute for Molecular Biophysics,

The Jackson Laboratory,

600 Main Street,

Bar Harbor, ME 04609, USA

e-mail: cremer@kip.uni-heidelberg.de sequence DYZ2 located within the heterochromatin region on human chromosome Yq12 was labelled with clone pHY2.1. Between 300 and 700 singleprobe molecules were resolved in individual chromatin domains, corresponding to a detected molecule density around $500 / \mu \mathrm{m}^{2}$, i.e., many times higher than resolvable by conventional fluorescence microscopy. A mean localisation accuracy of about $20 \mathrm{~nm}$ indicated a mean optical resolution in the $50 \mathrm{~nm}$ range. Beyond new perspectives for light microscopic studies of specific chromatin nanostructures, this may open a new avenue towards the general analysis of copy number of specific DNA sequences in small regions of individual interphase nuclei.

Keywords FISH - Chromatin nanostructure .

Optical resolution - Super-resolution - Localisation microscopy $\cdot$ Spectral precision distance microscopy (SPDM)

$\begin{array}{ll}\text { Abbreviations } \\ \text { DAPI } & 4^{\prime}, 6 \text {-Diamidino-2-phenylindol } \\ \text { DNA } & \text { Deoxyribonucleic acid } \\ \text { FISH } & \text { Fluorescence in situ hybridisation } \\ \text { FCS } & \text { Foetal calf serum } \\ \text { FWHM } & \text { Full width at half maximum } \\ \text { NA } & \text { Numerical aperture } \\ \text { PBS } & \text { Phosphate-buffered saline } \\ \text { PSF } & \begin{array}{l}\text { Point spread function } \\ \text { SPDM }\end{array} \\ & \begin{array}{l}\text { Spectral precision distance/position deter- } \\ \text { mination microscopy }\end{array} \\ \text { STED } & \text { Stimulated emission depletion }\end{array}$




\section{Introduction}

In recent years, nuclear genome structure has emerged as a central theme of cell biology (for reviews, see Lamond and Earnshaw 1998; Cremer and Cremer 2001; O’Brien et al. 2003; Misteli 2005, 2007; Rouquette et al. 2010). To study chromatin nanostructure, methods of ultrastructure analysis using ionising radiation, in particular electron microscopy, have proven to be a valuable tool for imaging beyond the conventional optical resolution of light microscopy, until recently thought to pose an absolute limit of about half a wavelength or $200 \mathrm{~nm}$ in the object plane and around $600 \mathrm{~nm}$ in the direction of the optical axis (Abbe 1873; Rayleigh 1896). In the recent past, however, a variety of laser-optical farfield microscopy techniques based on fluorescence excitation has been developed to overcome the 'Abbe-limit' of $200 \mathrm{~nm}$.

Some well-known methods are confocal 4PiLaser Scanning Microscopy (Cremer and Cremer 1978; Hell et al. 1994, 2003; Hänninen et al. 1995; Egner et al. 2002; Bewersdorf et al. 2006; Baddeley et al. 2006; Lang et al. 2010), structured/patterned illumination microscopy (Heintzmann and Cremer 1999; Gustafsson 2000; Baddeley et al. 2007; Schermelleh et al. 2008), STED microscopy (Hell and Wichmann 1994; Schrader et al. 1995; Hell 2007; Schmidt et al. 2008) or localisation microscopy techniques using far-field fluorescence microscopy (Cremer et al. 1996, 2010; Bornfleth et al. 1998; van Oijen et al. 1998; Edelmann et al. 1999, Edelmann and Cremer 2000; Esa et al. 2000; Lacoste et al. 2000; Schmidt et al. 2000; Heilemann et al. 2002; Betzig et al. 2006; Hess et al. 2006; Egner et al. 2007; Reymann et al. 2008; Lemmer et al. 2008). Using these techniques, an effective optical resolution in the 10 $20 \mathrm{~nm}$ regime has been obtained. For the first time, this enabled 'nanoimaging' of intracellular biostructures at macromolecular resolution using fluorescence excitation by visible light.

The basis of localisation microscopy as a far-field fluorescence microscopy 'nanoimaging' technique is the independent localisation of 'point like' objects excited to fluorescence emission by a focused laser beam or by non-focused illumination via 'optical isolation'; i.e. the localisation is assigned by appropriate spectral features ("signatures"), allowing focused, structured or homogeneous illumination schemes (Fig. 1).
Furthermore, a large variety of spectral signature modes can be used, including simple spectral absorption/emission characteristics, fluorescence life times and time-dependent phosphorescence/luminescence. This method, designated 'spectral precision microscopy' or spectral precision distance/position determination microscopy (SPDM) was conceived and realised in proof-of-principle experiments already in the 1990s (Cremer et al. 1996, 1999; Bornfleth et al. 1998; Rauch et al. 2000; Esa et al. 2000, 2001). Early 'proof-of-principle' experiments using confocal laser scanning fluorescence microscopy at room temperature to determine the positions $(x y z)$ and mutual Euclidean distances of small adjacent sites on the same large DNA molecule (translocation chromosome $t(9 ; 22)$ in human cell nuclei) labelled with three different spectral signatures yielded a lateral effective resolution of about $30 \mathrm{~nm}$ (ca. 1/16th of the wavelength used) and ca. $50 \mathrm{~nm}$ axial effective resolution (Esa et al. 2000): Sites with a 3D distance of only $50 \mathrm{~nm}$ were still discriminated; their $(x y z)$ positions were determined independently from each other with an error of a few tens of nanometers, including the correction for optical aberrations.

Compared with related ideas (Burns et al. 1985; Betzig 1995), in the SPDM approach, the focus of application was (1) to perform super-resolution analysis by the direct evaluation of the positions of the spectrally separated objects; (2) to adapt the method to far field fluorescence microscopy at temperatures in the $300 \mathrm{~K}$ range; (3) to specify spectral signatures to include all kinds of fluorescent emission parameters suitable, from absorption/emission spectra to fluorescence life times (Heilemann et al. 2002) or any other method allowing 'optical isolation' (Cremer et al. 2001).

In the last few years, approaches based on principles of SPDM and related methods have been considerably improved by several groups, especially by using photoswitchable fluorochromes. By imaging fluorescent bursts of single molecules after light activation with a first wavelength, using a second excitation wavelength the position of the molecules was determined with a precision significantly better than the full width at half maximum (FWHM, a parameter describing the width of a peak in a curve, in this case the microscopic Point Spread Function (PSF). The PSF is the normalised intensity distribution of a fluorescent point source; the FWHM of the 
PSF is a measure for the optical resolution at a given spectral signature). These microscopic techniques were termed photo-activated localisation microscopy (PALM) (Betzig et al. 2006), fluorescence PALM (Hess et al. 2006), stochastic optical reconstruction microscopy (Rust et al. 2006) or PALM with independently running acquisition (Geisler et al. 2007, Andresen et al. 2008). All these approaches use special fluorochromes which can be photoswitched between two different spectral states. Recently, we applied SPDM in the nanometer resolution scale to conventional fluorochromes that show reversible photobleaching, which is a general behaviour of several fluorescent proteins, e.g., CFP, Citrine or eYFP (Patterson and Lippincott-Schwartz 2002; Sinnecker et al. 2005; Hendrix et al. 2008). In such cases, the fluorescence emission of certain types can be described by assuming three different states of the molecule: A fluorescent state $M_{\mathrm{fl}}$, a reversibly bleached state $M_{\mathrm{rbl}}$ and an irreversibly bleached state $M_{\mathrm{ibl}}$. By illumination, some of the molecules are switched to $M_{\mathrm{rbl}}$ or to $M_{\mathrm{ibl}}$. The stochastical transfer of the molecules from $M_{\mathrm{rbl}}$ to $M_{\mathrm{fl}}$ and then to $M_{\mathrm{ibl}}$ can be used for optical isolation of the detected molecules and thus their positions can be determined. With velocity constants $k_{1}, k_{2}$ and $k_{3}$, the transition scheme can be assumed as follows

$$
M_{\mathrm{rbl}} \underset{k_{1}}{\stackrel{k_{2}}{\longrightarrow}} M_{\mathrm{fl}} \stackrel{k_{3}}{\longrightarrow} M_{\mathrm{ibl}}
$$

We demonstrated recently that reversible photobleaching can be used for super-resolution imaging (SPDM) of cellular nanostructures labelled with conventional fluorochromes such as Alexa dyes (Reymann et al. 2008; Baddeley et al. 2009) or fluorescent proteins (Lemmer et al. 2008, 2009; Gunkel et al. 2009). This effect may be obtained by appropriate physical conditions, such as an exciting illumination intensity in the $10 \mathrm{~kW} / \mathrm{cm}^{2}$ to several $100 \mathrm{~kW} / \mathrm{cm}^{2}$ range; only one exciting wavelength is necessary both for activation and read out of a given molecule type. Under these conditions, a large number of images or frames is taken rapidly one after the other (typical frame rates around $20 \mathrm{fr} / \mathrm{s}$ or more, up to a total of 1,000 to 4,000 frames); in each frame, under appropriate conditions the distance between the diffraction images of individual fluorescent molecules is typically larger than the 'Abbe limit', i.e., larger than the FWHM of the PSF of the optical system used. Recently, a related technique (Fölling et al. 2008) has been described for several other dyes like Atto532, Citrine or PhiYFP called ground state depletion microscopy followed by individual molecule return.

In the present study, we combined SPDM with fluorescence in situ hybridization (FISH)-labelling to quantitatively analyse the spatial distribution of specifically FISH-labelled DNA sequences in small regions of human cell nuclei. Heterochromatin region Yq12 was labelled with a cloned DYZ2 fragment (pHY2.1, Cooke et al. 1982) in human fibroblast nuclei. Repetitive DNA sequence DYZ2 is a major component of the heterochromatic region on human Yq12 and comprises approximately $20 \%$ of the Y chromosomal DNA (Cooke 1976). Arranged in tandem arrays, the $2.47 \mathrm{~kb}$ repeat is distributed in about 2,000 copies over the entire length of Yq12 (Schmid et al. 1990).

\section{Materials and methods}

Cell culture and specimen preparation

Human diploid fibroblast cells VH7 (kindly provided by Prof. Dr. Beauchamp from DKFZ, Heidelberg) were routinely cultivated in Dulbecco's Modified Eagle medium supplemented with $10 \%$ FCS and penicillin/streptomycin at $37^{\circ} \mathrm{C}$ in a humidified $\mathrm{CO}_{2}$ incubator. For specimen preparation, cells were seeded onto cover slips, grown until $70-80 \%$ confluency and subsequently fixed with $4 \%$ formaldehyde in PBS. Permeabilisation steps included treatment in $0.5 \%$ Triton-X in PBS, $20 \%$ glycerol in PBS, repeated freezing-thawing in liquid nitrogen and incubation in $0.1 \mathrm{~N} \mathrm{HCl}$, according to standard protocols for the preparation of 3D preserved nuclei (Bridger and Lichter 1999; Solovei et al. 2002a). Cover slips were stored in $50 \%$ formaldehyde in $2 \times \mathrm{SSC}(\mathrm{pH} 7)$ at $4^{\circ} \mathrm{C}$ until usage.

\section{Probe preparation}

Clone pHY2.1 (Cooke et al. 1982), composed of the $2.1 \mathrm{~kb}$ HaeIII fragment inserted into plasmid pBR328 (a kind gift from Dr. G. Rappold, Institute of Human Genetics, University of Heidelberg) was labelled by standard nick translation using AlexaFluor ${ }^{\circledR} 568$ 
(Invitrogen, Germany) as fluorescent dye. Labelled DNA was co-precipitated with salmon sperm DNA serving as carrier DNA and resuspended in hybridisation buffer consisting of $50 \%$ deionised formamide, $10 \%$ dextran sulphate in $4 \times \mathrm{SSC}$.

\section{FISH and immunocytochemistry}

Probe DNA was denatured at $78^{\circ} \mathrm{C}$ for $7 \mathrm{~min}$. Cellular DNA was denatured in $70 \%$ formamide in $2 \times \mathrm{SSC}$ (pH 7) at $72^{\circ} \mathrm{C}$ for $2 \mathrm{~min}$. Subsequently, the denatured probe was placed onto a cleaned slide and the cover slips carrying the cells were lifted onto it. To prevent drying, cover slips were sealed with rubber cement. Hybridisation was performed in a humidified chamber at $37^{\circ} \mathrm{C}$ for 2 days. Post-hybridisation washings were done in $4 \times \mathrm{SSC} /$ Tween $20(0.2 \%)$ at $42^{\circ} \mathrm{C}$ and $1 \times \mathrm{SSC}$ at $60^{\circ} \mathrm{C}$. For the analysis of the cell cycle, a mouse monoclonal antibody against nuclear protein pKi67 (Dako, Denmark) was applied. pKi67 displays differential patterns according to cell cycle stage (Gerdes et al. 1983). Detection was done with an Alexa Fluor ${ }^{\circledR} 488$-conjugated goat anti-mouse antibody (Invitrogen, Germany). Cells were mounted in ProLong ${ }^{\circledR}$ Gold antifade reagent (Invitrogen, Germany) and sealed with nail polish.

\section{Data registration}

For localisation microscopy, a microscope system was used allowing spatially modulated illumination with three laser lines (Baddeley et al. 2007). For the measurements reported here, this instrument was used in the single objective lens mode. A schematic of the microscopy setup is shown in Fig. 2.

The setup is equipped with three laser sources providing laser light at $\lambda=488 \mathrm{~nm}, \lambda=568 \mathrm{~nm}$ and $\lambda=647 \mathrm{~nm}$ (Lexel Laser, USA). Lenses L2 and L3 (Linos Photonics, Germany) expand the laser beam to a diameter of approximately $20 \mathrm{~mm}$. A 50:50 beam splitter (Edmund Optics, Germany) evenly distributes the beams onto the two interferometer arms. The left optical path provides wide-field illumination: The beam is directed to the objective (O1), passing through an achromatic lens (L4) and a mirror (S2), resulting in collimated illumination of the sample, positioned between the two objectives. Currently in use are $100 \times$ oil objectives with an NA of 1.4 (Leica, Germany). Movement of the sample is provided by a 3-axis stepper motor stage and a 2-axis piezo stage (Physik Instrumente, Germany). The right optical path is utilised for localisation microscopy: In addition to an achromatic lens (L5) focusing the beam into the back focal plane of the objective $(\mathrm{O} 2)$, a second lens (L3) provides the higher laser illumination intensities needed for this type of localisation microscopy. For the illumination of dyes, intensities were in the order of $10 \mathrm{~kW} / \mathrm{cm}^{2}$. The emission light from the sample is collected by the right hand objective lens (O2), passes through the dichroic mirror D4 (AHF, Germany) reflecting the laser lines, and is focused by a $\times 1$ tube lens (Leica, Germany) onto the CCD chip of a highly sensitive camera (PCO Imaging, Germany). Remaining laser light and out of band fluorescence are blocked by blocking filter BF (AHF, Germany). A typical time series encompassing 1,000 2D images (frames) was acquired with a repetition rate between 10 and $16 \mathrm{~Hz}$.

Data evaluation

In localisation microscopy, the essential concept to surpass the resolution limit of conventional light microscopy is the optical isolation of single fluorescent molecules by using appropriate spectral signatures, which may include photo-physical or photo-chemical properties of the fluorescent dye molecules (e.g., photoswitching or photoconversion). This means that at any given time, the distance between two molecules of the same spectral signature has to be larger than the conventional optical resolution (FWHM of the PSF). In conventional microscopy, on the contrary, the image of the fluorescence-labelled object emerges from the simultaneous detection of all fluorescent molecules, i.e., also from molecules with a distance smaller than the conventional resolution: in this case, due to diffraction, the object appears blurred and the resulting loss of structural information cannot be reversed. In localisation microscopy, however, a time series of only a few single molecules per image (i.e., with distances larger than the conventional resolution) is acquired. The result is a large number of single images or frames of sparsely distributed molecules with non-overlapping diffraction patterns. This allows to determine the positions of the individual fluorophores with a precision mainly depending on the detected photon numbers (Bornfleth et al. 1998; Edelmann and Cremer 2000; Thompson et al. 2002). All positions obtained from the 

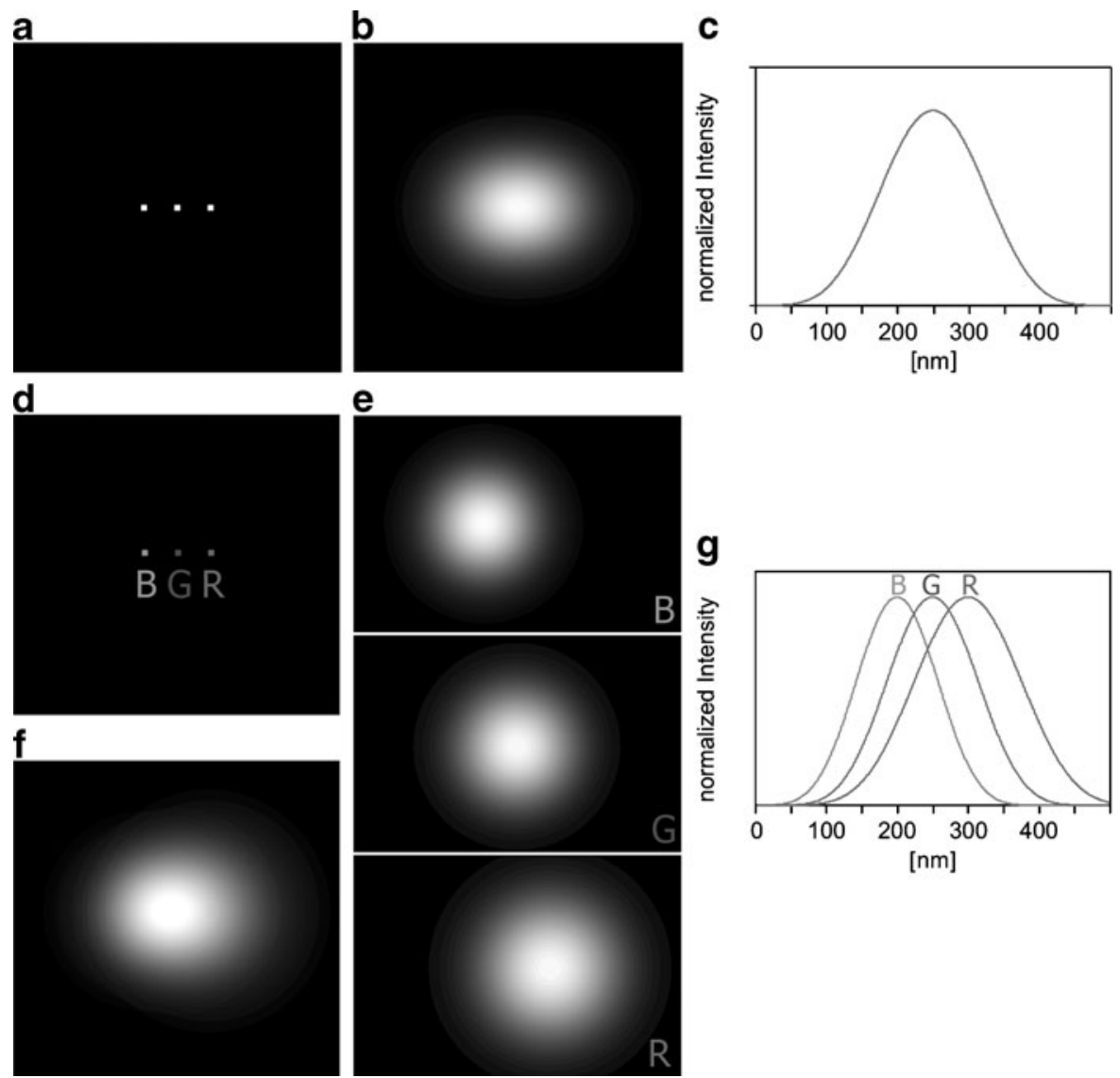

Fig. 1 Principle of SPDM. a, d Three point-like objects are located in the $x y$ plane within $50 \mathrm{~nm}$ distance to the middle one. They are labelled with the same spectral signature in (a), or with three different, unique spectral signatures in (d). The computed "virtual microscopy" responses of the objects in (a) and (d) (parameters for computation are NA=1.4 and system magnification of $\times 63$ ) are shown in (b), (e) and (f). In (f), the

individual frames are assigned to the same 'localisation map'. Thus, an image of the measured object (positions of all molecules detected) can be reconstructed that is not limited by diffraction.

The individual steps for the position determination of a single fluorophore are as follows:

1. Segmentation of the signal within the wide-field data

2. Fitting of an appropriate model function to the data to determine the individual molecule positions.

For the detection and segmentation of the signals, established algorithms such as CLEAN (Högbom 1974) can be used. different spectral components are imaged together whereas in (e), the same signals are recorded independently from each other. Normalized linescans through the objects in (b) and (e) are shown in (c) and (g). Figures from (Kaufmann et al. 2009) adapted from (Cremer et al. 1999), based on the SPDM concept of (Cremer et al. 1996)

As a model function $f(x, y)$ for the PSF, a $2 \mathrm{D}$ gaussian is approximated using a linear estimate of the background noise. Thus, the model function is

$$
\begin{aligned}
f(x, y)= & p_{1} \exp \left(-\frac{\left(x-p_{2}\right)^{2}+\left(y-p_{3}\right)^{2}}{2 p_{4}^{2}}\right)+p_{5} \\
& +p_{6}\left(x-p_{2}\right)+p_{7}\left(x-p_{3}\right)
\end{aligned}
$$

where $p_{1}$ to $p_{7}$ are, respectively, the signal amplitude, the $x$ and $y$ coordinates of the barycenter (gravity center of fluorescence intensity), the standard deviation as a measure of the spread of the signal, the constant contribution of background noise and the 


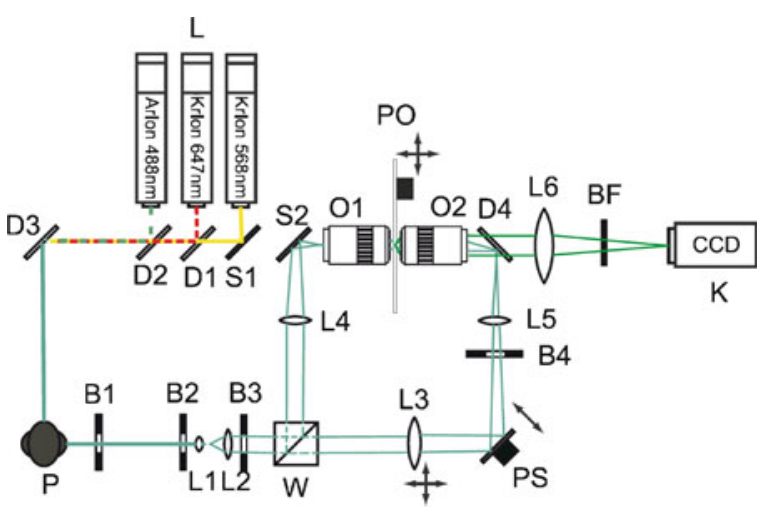

Fig. 2 Experimental setup used for localisation microscopy. D1-D4 dichroic elements, $L$ lasers, L1-L6 lenses or lens systems, $B 1-B 4$ field diaphragm, $W$ beam splitter, O1-O2 objective lenses, $P S$ piezo translation stage, $P O$ piezoelectrical stage, $S 2$ mirror, $B F$ blocking filter, $P$ periscope, $K \mathrm{CCD}$ camera. The appropriate laser intensities required are gained by adjusting lens L3. For a detailed description, see text linear approximation of the background signal in the $x$ and $y$ directions. By using this model function with appropriate parameters, signals above and below the object plane are excluded, i.e., an 'optical section' of about $600 \mathrm{~nm}$ thickness is obtained. For further details on the localisation algorithm, please refer to Kaufmann et al. (2009) and Lemmer et al. (2009). The algorithms used for data evaluation of the localisation microscopy images were implemented in MATLAB (7.0.1, The MathWorks, Natick, USA). In addition, measurements on the segmented data were performed using the DipImage toolkit (http:// www.ph.tn.tudelft.nl/DIPlib/).

The simulation in Fig. 3 illustrates the basic concept of localisation microscopy with emphasis on the relationship between the localisation accuracy and the number of detected photons.
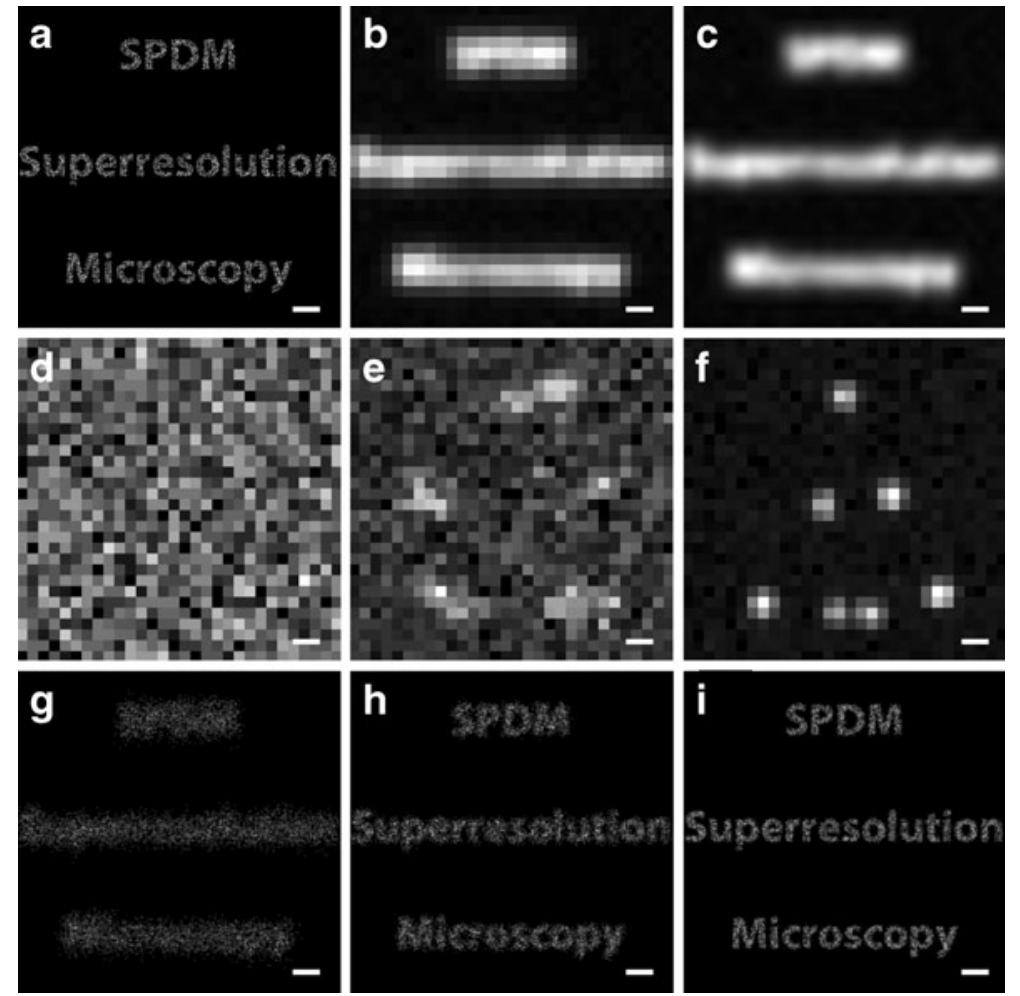

Fig. 3 Virtual localisation microscopy (computer simulation). Upper panel: a simulated structure, b simulated wide-field image, $\mathbf{c}$ interpolated image of (b). Middle panel: Simulated wide-field images of a small number of individual single molecule signals differing in detected photon number; the level of background noise remains constant: In (d), the average number of detected photons/molecule assumed was $N_{\text {phot }}=15$, in (e) $N_{\text {phot }}=100$ and in (f) $N_{\text {phot }}=1,500$. Lower panel: Virtual localisation microscopy of the simulated structure given in (a) with varying localisation accuracies (standard deviations): In (g), it was $\sigma=50 \mathrm{~nm}$, in (h) $\sigma=20 \mathrm{~nm}$ and in (i) $\sigma=10 \mathrm{~nm}$. The size of the scale bars is $250 \mathrm{~nm}$. Note that the effective pixel size in the individual images differs. For the simulated widefield images in (b) and (d-f) it is $100 \mathrm{~nm}$, whereas in (a, c) and $(\mathbf{g}-\mathbf{i})$, it is $5 \mathrm{~nm}$ 

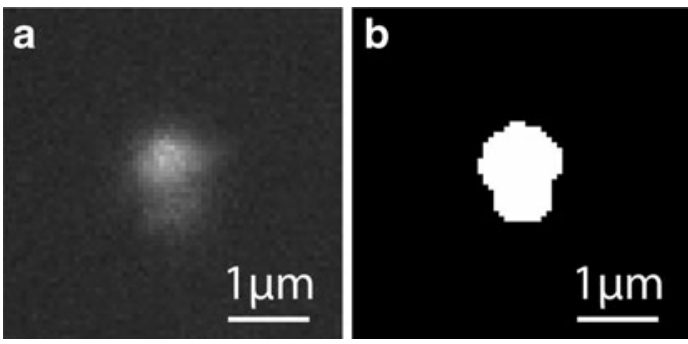

Fig. 4 Threshold-based segmentation. a Wide-field image of the labelled region, b segmented image obtained by morphological closing, c interpolated wide-field image and the

In Fig. 3(a), the simulated structure and the positions of the individual fluorophores are shown. The total number of fluorescent molecules amounts to 9,289 , and the area covered by the object is $0.77 \mu^{2}$, resulting in a fluorophore density of
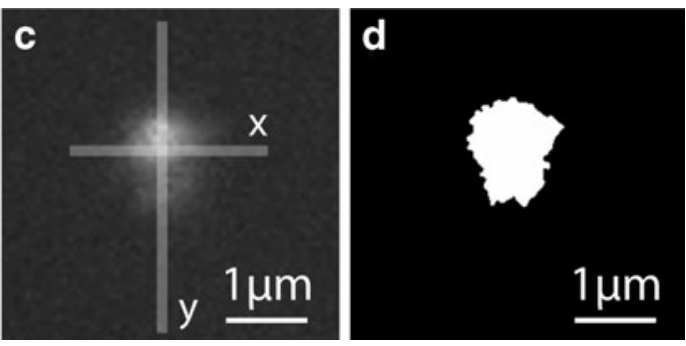

corresponding segmented image (d). Pixel sizes in (a, b) are 65 and $13 \mathrm{~nm}$ in (c, d). The cross in (c) indicates the axes along which the line profiles in Figs. 5 and 12 are drawn

$12,060 / \mu \mathrm{m}^{2}$. Figure 3(b) shows the blurred diffraction limited image as simulated for wide-field microscopy. The accompanying interpolated image is depicted in Fig. 3(c). The wide-field images were simulated assuming a wavelength of $500 \mathrm{~nm}$, an NA
Fig. 5 Intensity profiles for the structure shown in Fig. 4 in $x$ (a) and $y$ (b) direction (indicated by the axes in Fig. 4(c))

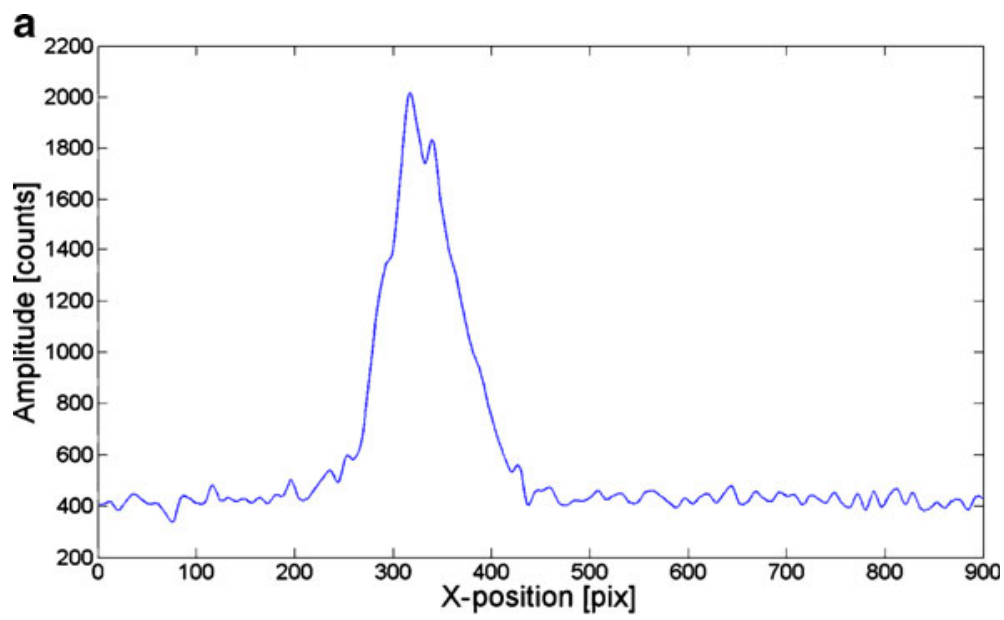

b

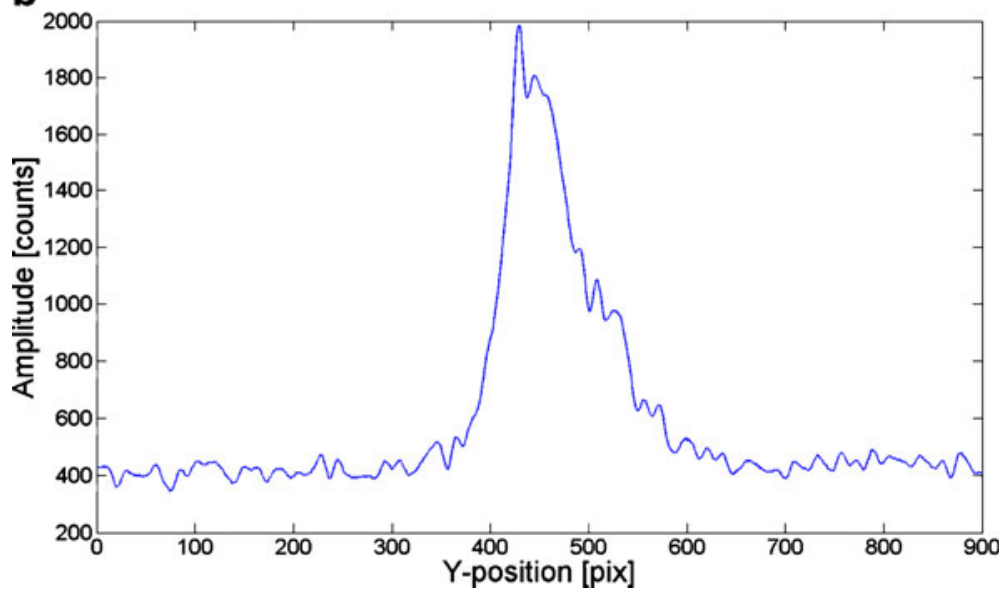


of 1.46 and 150 detected photons on average per fluorophore. This choice of parameters almost resembles ideal conditions and admittedly is rather optimistic. For the convolution of the simulated structure (a), the Airy disc was utilised as intensity PSF. The Airy disc is the image of an infinitely small fluorescent object in the focal plane of a perfect optical system (Rayleigh 1896). Its rotational symmetric intensity distribution follows from the equation

$I(r, \lambda)=I_{0}\left(\frac{2 J_{1}(2 \pi N A r / \lambda)}{2 \pi N A r / \lambda}\right)^{2}$

where $J_{l}$ is the first order Bessel function and $r$ is the distance to the centre in $\mathrm{nm}$.

The middle panel of Fig. 3 shows the effect of detected photon numbers per signal on localisation accuracy, assuming equal background levels in each case. For the PSF, the function described above was used. In (d), the photon number $N_{\text {phot }}$ of the

a
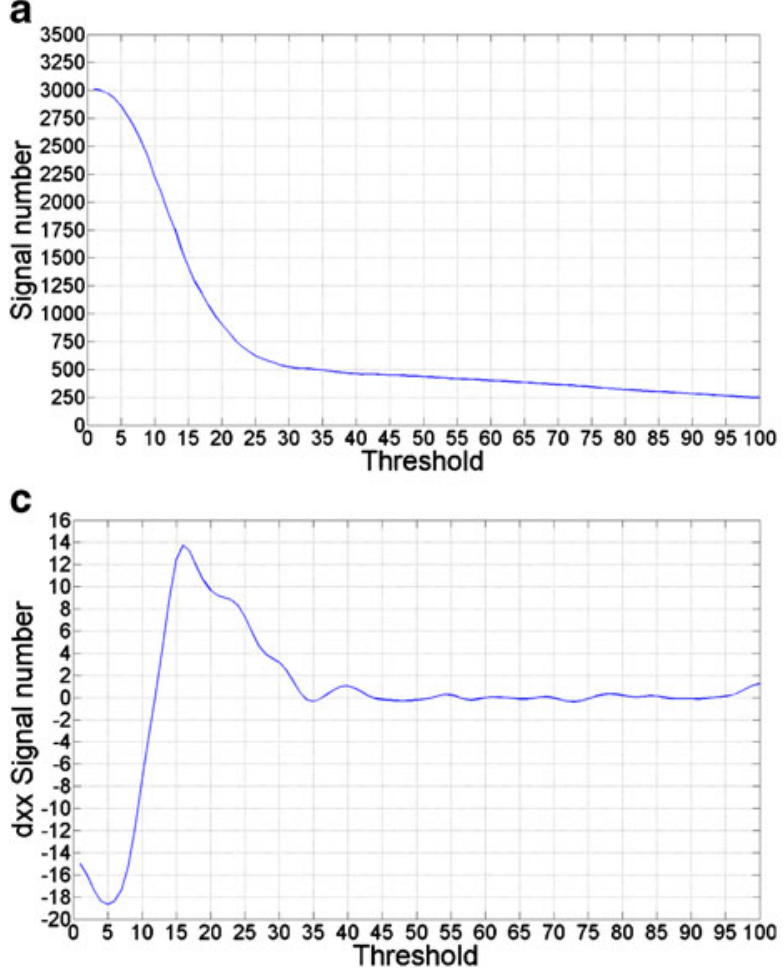

Fig. 6 Principle of the automated thresholding procedure. a Number $(n)$ of remaining molecules as a function of the number of neighbours within a defined vicinity designated as threshold. In (b) and (c), the first and the second derivatives are shown, fluorescence signal of active molecules averages to 15 , in (e) to 100 and in (f) to 1,500 photons. For each molecule, a variation in detected photon number, expressed as standard deviation of a normal distribution, of $30 \%$ of the mean was assumed. In (d), it is not possible to differentiate between background and signal. In (e), signals become recognisable but the precise number of molecules cannot be determined. In (f), the number and the position of the individual signals can be readily identified.

The effect of localisation accuracy on the attainable resolution is illustrated in the lower panel. For clarity, the corresponding images of the middle and lower row are simulated with consistent average photon numbers, i.e., in $(\mathrm{g})$, it averages to 15 , in (i) to 100 and in $(\mathrm{h})$ to 1,500 photons. In (g), a localisation accuracy of $50 \mathrm{~nm}$ along with the detection of a few tens of photons provided no additional structural information about the object, when compared to the conventional microscope image shown in (b). By the

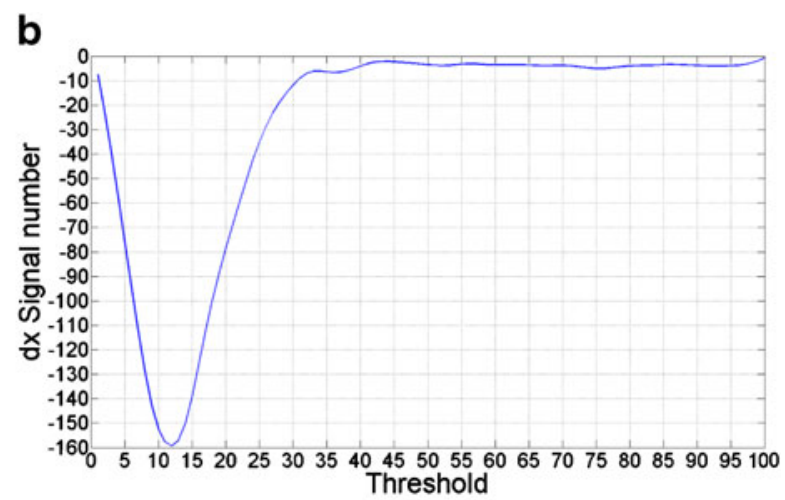

respectively. Both were determined by mathematical differentiation, of the curve in (a). Note the different scaling of the ordinate in each case 
detection of around 100 photons/molecule and a resulting localisation accuracy of $20 \mathrm{~nm}$, substructures become distinguishable (h) which are certainly not observable in the conventional wide-field image. The detection of more than 1,000 photons (i) is sufficient for a localisation accuracy better than $10 \mathrm{~nm}$. In combination with an adequate number of detected molecules, this allows a good reconstruction of the given nanostructure.

One of the fundamental quantitative parameters of a fluorescent chromatin nanostructure is its overall size (area). This can be done in different ways, e.g. by adapting appropriate model functions (Baddeley et al. 2010) or by interpolation. Figure 4 illustrates the impact of an interpolation procedure on the result of the segmentation process. Both raw data of an experimental wide-field image of a FISH signal (4a) and the accompanying interpolated image data (4c), obtained by a standard procedure of image processing termed "lossless interpolation" were segmented with a conventional threshold based method. The same threshold value was used in both cases. As a result of the interpolation procedure, the number of pixel within the images increases and the effective pixel size decreases accordingly. For Fig. 4(a), the pixel size is $65 \mathrm{~nm}$, and $13 \mathrm{~nm}$ for Fig. 4(c). Provided the image is Nyquist sampled (i.e., the pixel size is chosen in such a way, that the smallest possible structural component of the object is sampled at least 2 -fold), the information of all intermediate values is available and can be used as additional landmarks in the segmentation procedure. The spaces between pixels can thus be filled while all morphological information is retained.

The area of the labelled DNA region $(b, d)$ is extracted from the wide-field images $(a, c)$ by an operation from the field of mathematical morphology termed morphological closing (Matheron 1975; Serra 1982). As structuring element, a circular disc in the plane was utilised. Basically, this results in the elimination of small holes and the filling of gaps in contours. In addition, a certain amount of smoothing on an object contour is effected.

Without interpolation, the area of the segmented region is calculated to $0.942 \mu \mathrm{m}^{2}$ and with interpolation to $1.049 \mu^{2}$. This means the area is $11.3 \%$ larger in the interpolated image. It is easily observable that the non-interpolated images (Fig.4 a, b) appear rather pixelated, whereas the images subjected to interpolation are smoother to some extent, and the edges are shown in far more detail, thus allowing the extraction of considerably more structural information. For this,
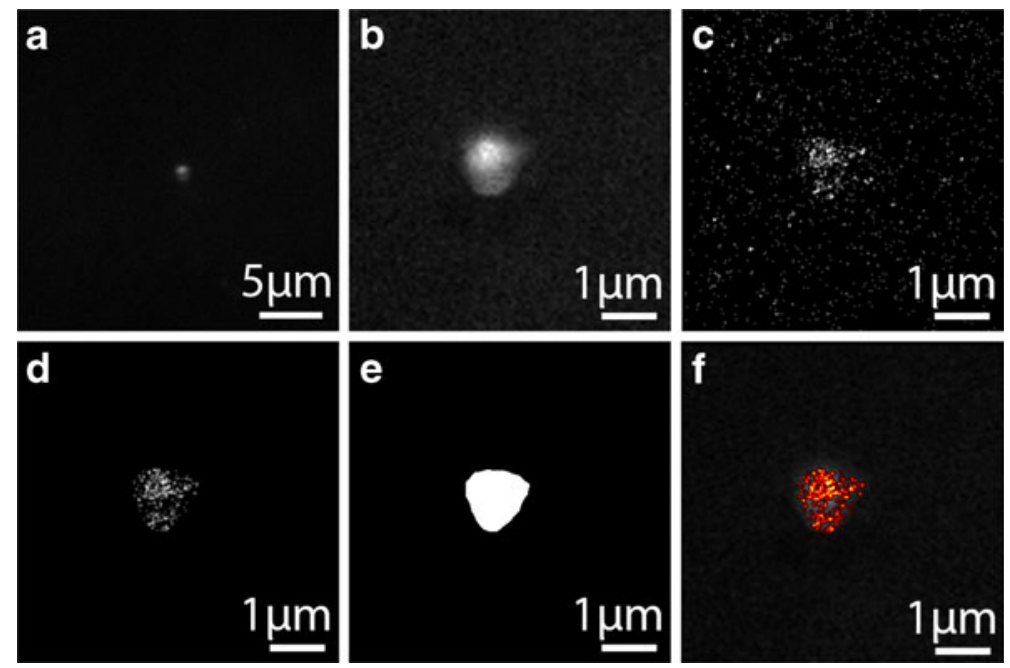

Fig. 7 Representation of the data processing procedure for a typical experimentally obtained FISH signal of DNA probe pHY2.1. a Wide-field image at low magnification; b corresponding interpolated magnification; c reconstruction of the localisation microscopy (SPDM) data, blurred with a Gaussian corresponding to the mean localisation accuracy; d segmented image of the SPDM data in (c); e binary image of the area obtained by morphological closing of the SPDM image in (d); $\mathbf{f}$ overlay of the image of the segmented region as seen in (d), displayed as a "heat map" encoding the molecule density, and the wide-field image (gray scale) shown in (b) 
however, a reliable discrimination of object and background and thus a good signal-to-noise ratio is important.

In Fig. 5, the line profiles, denoted in Fig. 4(c) are presented, indicating the excellent signal-to-noise ratio of the FISH-labelled chromatin structures.

Localisation microscopy, as every other fluorescence microscopical technique, is affected by non-specific labelling. For a correct quantitative evaluation of the chromatin nanostructure images obtained by SPDM, it is necessary to eliminate such non-specific signals from further consideration. For the investigation of the FISH-labelled regions, differences in the local fluorophore density were used to distinguish between specifically and nonspecifically bound dye molecules. The employed automatic segmentation algorithm determined the number of neighbours in a defined vicinity for the individual molecules and used these numbers subsequently as "density threshold" values (s): The higher $\mathrm{s}$, the lower the number $(n)$ of remaining molecules. Figure 6 shows the principal approach: On the assumption that the fluorophore density is significantly higher within the labelled structure than in the surrounding area, the total number $(n)$ of remaining molecules will decrease rapidly with increasing density threshold (s); this decrease will slow down considerably when all non-specifically bound molecules are eliminated (Fig. 6a). To obtain a quantitative, objective measure for the density threshold $\left(\mathrm{s}_{0}\right)$, derivative functions were applied. Figure 6(b) shows the first derivative, i.e., the alteration of the number of remaining molecules depending on the applied threshold value $\left(f^{\prime}(n)=\frac{d n}{d s}\right.$, where $n$ is the number of remaining molecules and $s$ the density threshold value). This curve first falls down rapidly to a minimum and then rises versus zero values (i.e. there $\mathrm{n}$ changes only slightly with increasing density threshold values s). To determine the density threshold $\mathrm{s}_{0}$ where the decline of $(n)$ starts to slow down, the first derivative (Fig. 6b) was differentiated again mathematically to obtain the second derivative of the curve in Fig. 6(a). Accordingly, $f^{\prime \prime}(n)=\frac{d^{2} n}{d s^{2}}$ was calculated and plotted in Fig. 6(c). The density threshold value at the second zero crossing of the second derivative was then used as a measure for $s_{0}$. The entire process was established as an automatic algorithm and subsequently used for the segmentation of the localization microscopy images of the FISH-labelled chromatin regions.
Fig. 8 Selection of typical experimentally obtained FISH signals for DNA probe pHY2.1 and the corresponding processed data. Columns from I to IV: $I$ wide-field images of the FISH signal; II segmented SPDM images; III binary images of the areas in (II); $I V$ wide-field images of pKi67 indicating cell cycle phase and the nuclear boundary

Figure 7 outlines the entire data processing procedure as applied in this study using an experimentally obtained FISH signal. Prior to the localisation measurements a conventional wide-field image is obtained (Fig. 7a), providing an overview of the imaged region. The corresponding interpolated magnification is shown in Fig. 7(b). The reconstructed localisation image shown in Fig. 7(c) is subjected to the automatic segmentation procedure outlined above (Fig. 6); the result is presented in Fig.7d. Morphological information of the labelled region is extracted from the binary image presented in Fig. 7e. Figure 7(f) depicts the good agreement of the image of the segmented region and the wide-field image. In this case, the segmented region is represented as a "heat map": The colour encodes the density of the detected molecules; the wide-field image is displayed in gray scale.

\section{Results}

Localisation microscopy (SPDM) was performed on the heterochromatic region on Yq12 labelled with Alexa568 dye in 25 human diploid fibroblast cell nuclei and evaluated using appropriate algorithms as described above. As an indication of cell cycle phase, nuclear protein pKi67 was detected by Alexa488 coupled antibodies. In Fig. 8, two galleries of typical data resulting from a localisation microscopy experiment are shown. In each case, the wide-field image of the chromatin region, the segmented SPDM image, the binary image of the segmented SPDM image and the wide-field image of nuclear protein pKi67, indicating the cell cycle phase are shown. Both wide-field images were acquired at low illumination intensities before the localisation measurements. From the distribution pattern of pKi67 (Gerdes et al. 1983) nuclei in Fig. 8(a-f) and Fig. 8(g-i) were assigned to G1/S phase, whereas nuclei in Fig. 8(j-1) were assigned to $G 2$. 


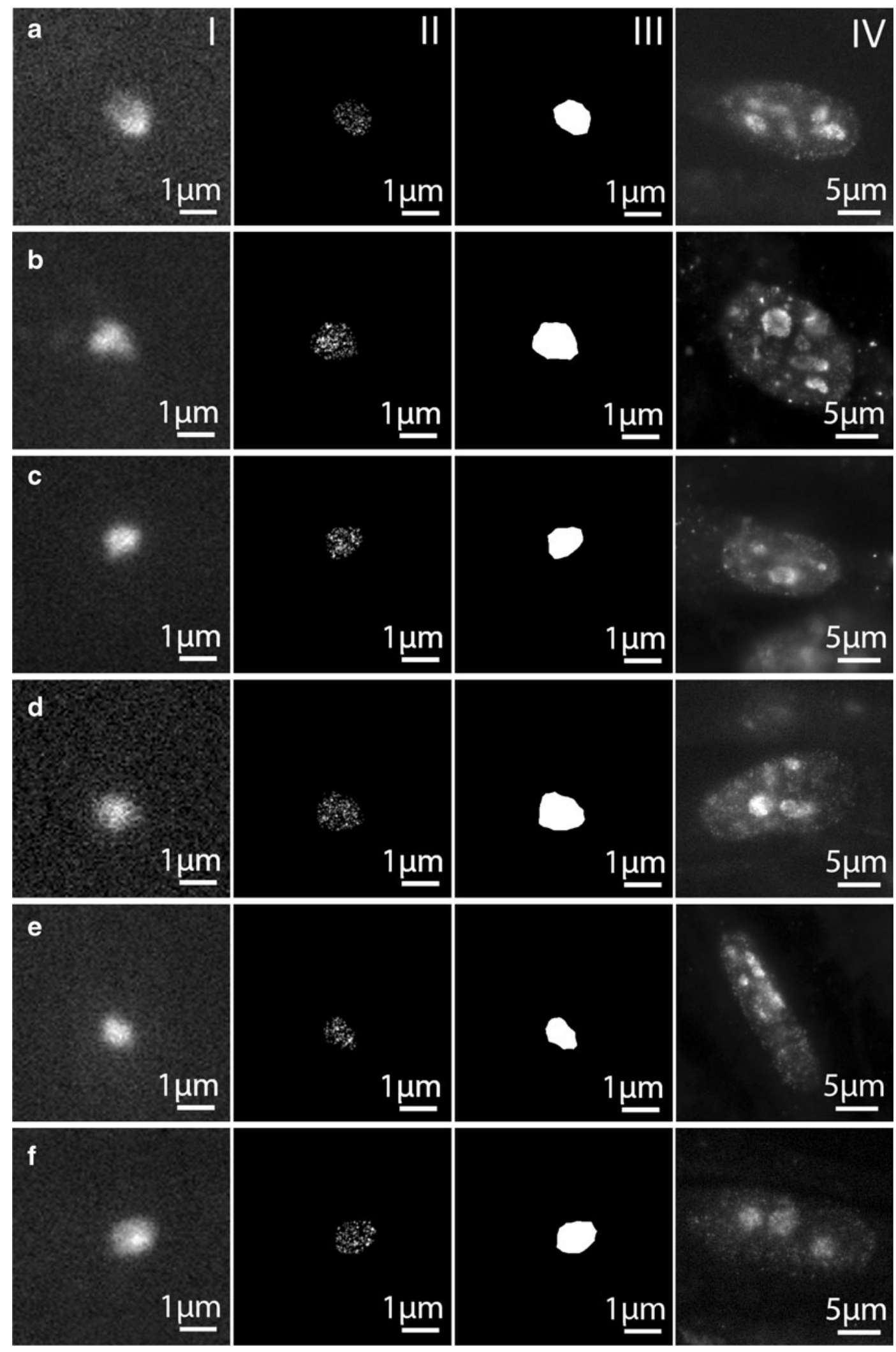




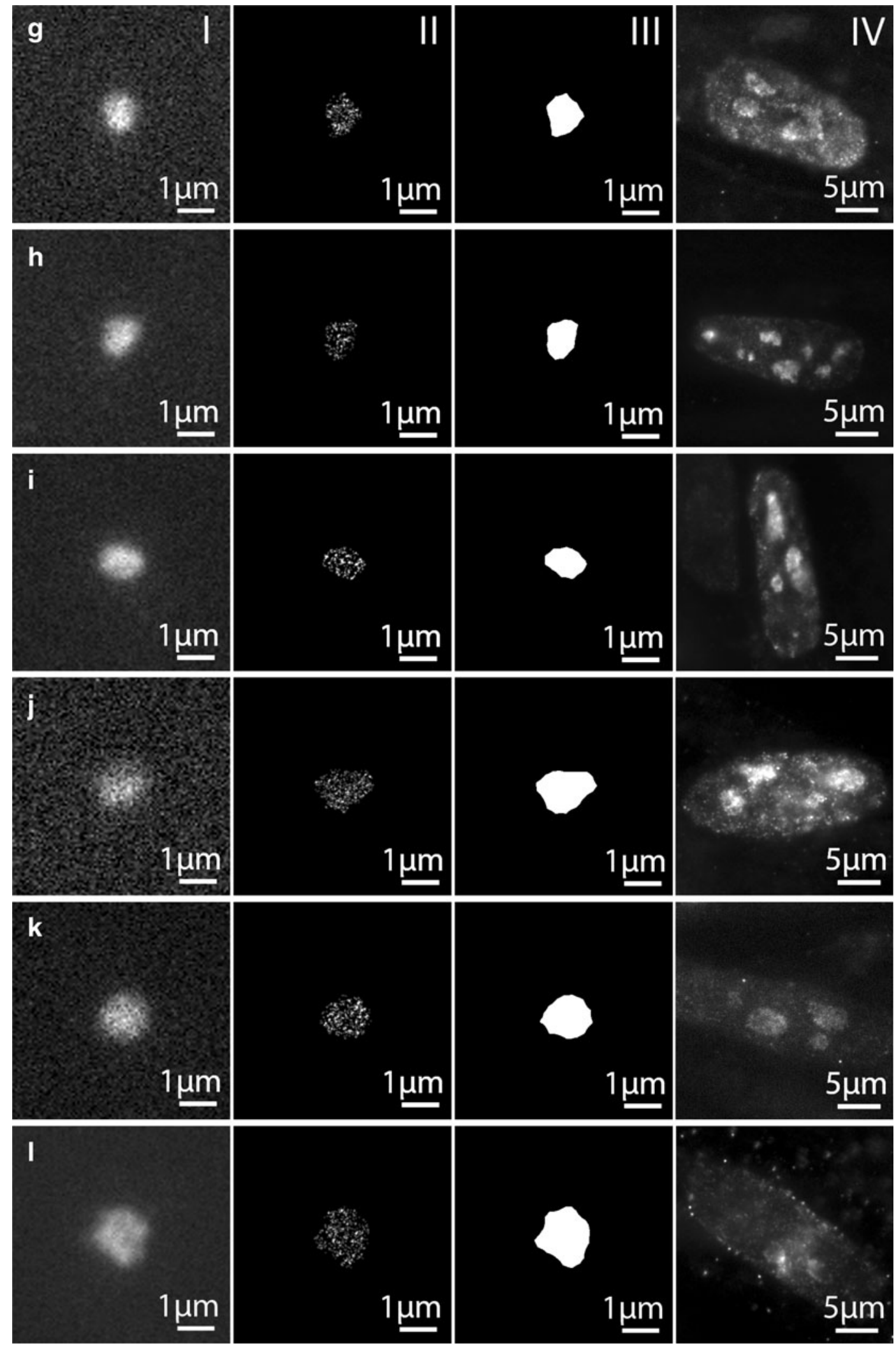


Fig. 8 (continued)

The quantitative, automated evaluation procedures applied allowed the extraction of various structural parameters such as variations in size, shape, and signal number. For a description of the shape, we calculated for every FISH signal the area, extent and anisotropy. The anisotropy can be defined as the ratio between the spread along the major and minor axes. In Fig. 9, the histograms of the segmented areas of the chromatin region (a) and the number of single molecule signals within these areas are shown (b).

Both histograms display several maxima at various positions. The analysis of the area yielded values ranging from 0.50 to $1.65 \mu \mathrm{m}^{2}$ and a mean value of $0.86 \pm 0.28 \mu \mathrm{m}^{2}$. The number of signals was found to range from 281 to 713 with an average of $443 \pm 114$. The comparison of FISH labelled area and cell cycle stage (as determined from the pKi67 pattern) indicates that all nuclei with areas smaller than $1.1 \mu^{2}$ are in G1/S phase.

The scatter diagrams in Fig. 10 depict the relationship between different structural parameters.

In Fig. 10(a), the number of detected signals was plotted against the area of the chromatin regions. The basic trend of the graph shows that the number of signals increases linearly with the area. Figure 10(b) displaying the anisotropy of the chromatin region reveals a strong linear relationship with a slope of 0.972. This suggests that on average the DNA regions do not show a shape deviating considerably from a sphere.

Another possibility to obtain structural information from the SPDM data is to utilise the position data directly. This eliminates the necessity to determine intensity thresholds as in conventional epifluorescence microscopy segmentation. As a measure for the extension of the FISH-labelled region, the spread of the molecule positions along each of the image axes is used. To quantify the spread, the standard deviation of the individual signal coordinates in the $x$ and $y$ directions was used. First, the position of the barycenter $P(x, y)$ of the individual molecule positions of the segmented SPDM images was determined by

$\bar{x}=\frac{\sum_{i} x_{i}}{N}$ and $\bar{y}=\frac{\sum_{i} y_{i}}{N}$

where $N$ is the total number of signals. Then the spread of the molecule distances $(X, Y)$ to the respective barycenters was determined. The basic principle was adapted from the Multi point model for the evaluation of conventional confocal data (Baddeley et al. 2010).

As an example, Figure $11(a, b)$ shows the histograms of the $X$ and $Y$ positions of the combined single molecule signals obtained for the FISH signal depicted in Fig. 4. As expected from a structure with many molecules positioned at different distances to the barycenter, a broad distribution was obtained. a

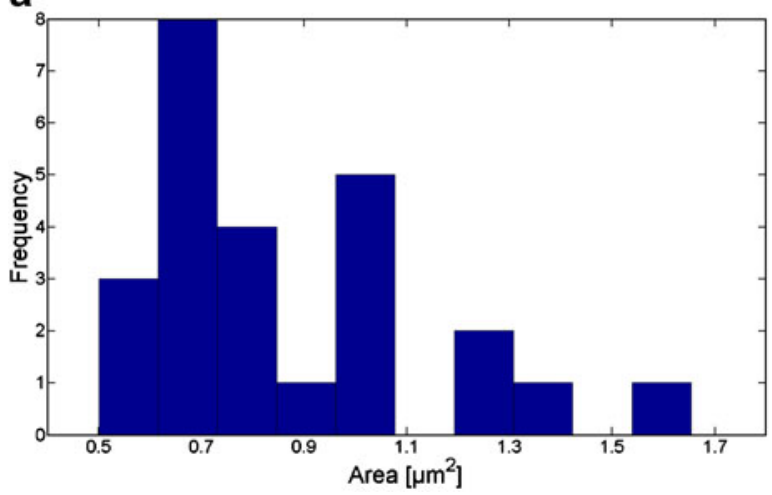

b

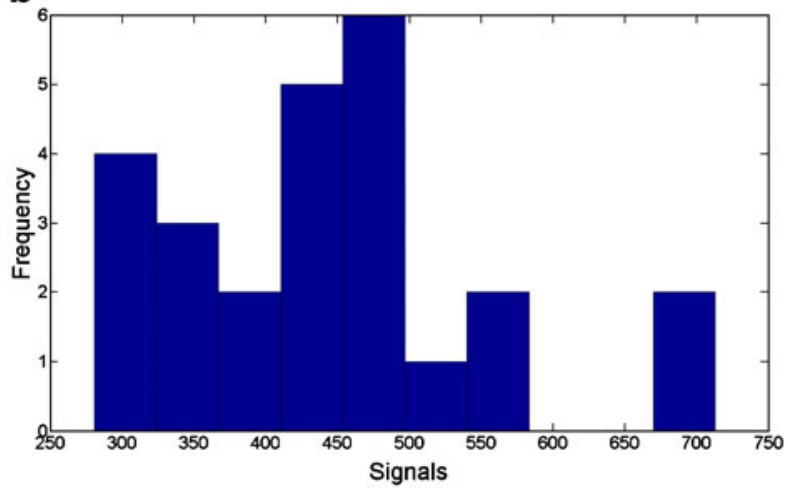

Fig. 9 Distributions of a the segmented areas of the FISH signals and $\mathbf{b}$ the number of detected molecules obtained per FISH signal 


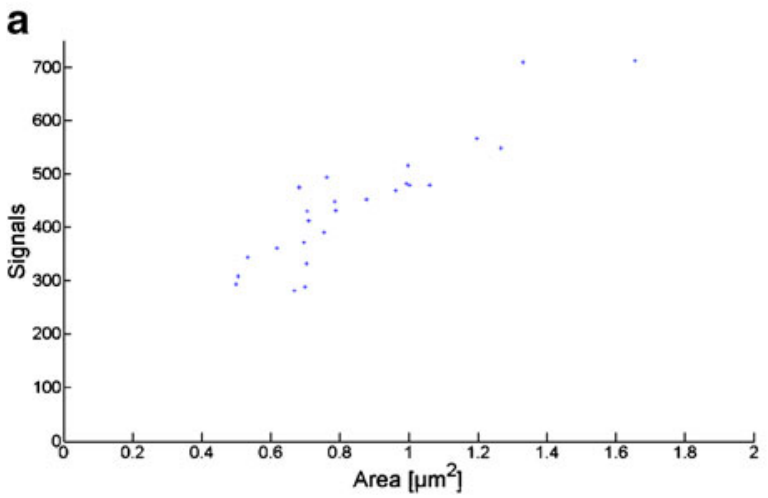

Fig. 10 Scatter diagrams displaying in a the relationship between the number of detected single molecule events and the area of the chromatin regions and in $\mathbf{b}$ the anisotropy of the

Whether the frequency variations indicate a specific substructure remains to be elucidated. The same data as in Fig. 11(a, b) are presented in Fig. 11(c, d) as quantile plots (i.e., accumulated frequency histograms). On the ordinate the distances $(X, Y)$ of the signal to the respective barycenter is given as a function of the part of the distribution; i.e., every value of the distribution is plotted against the proportion of all smaller values. From this, in the $x$ direction, a medium value of $495 \mathrm{~nm}$ was obtained while in the $y$ direction, the medium value was $405 \mathrm{~nm}$. This suggests an anisotropy of 495/405= 1.22 .

In Fig. $12(a, b)$, the distributions of the standard deviations in the $x$ and $y$ directions measured for the positions of all the molecules detected in the FISHlabelled regions for all 25 nuclei analysed are presented. In order to obtain a value that can be compared to other size measurements, the individual values can be converted to the FWHM of a Gaussian distribution by multiplication with a factor of 2.35 .

In contrast to Fig. 9, displaying the distribution of segmented areas for all 25 nuclei, the distributions of the standard deviations in Fig. 12 appeared to be more homogeneous, suggesting a bimodal distribution of nuclei with standard deviations smaller than $300 \mathrm{~nm}$ and of nuclei with standard deviations larger than $300 \mathrm{~nm}$. It may be noted that the nuclei with a FISHlabelled Yq12 area larger than $1.1 \mu^{2}$ (Fig. 9) are represented by standard deviations larger than $325 \mathrm{~nm}$ in both directions.

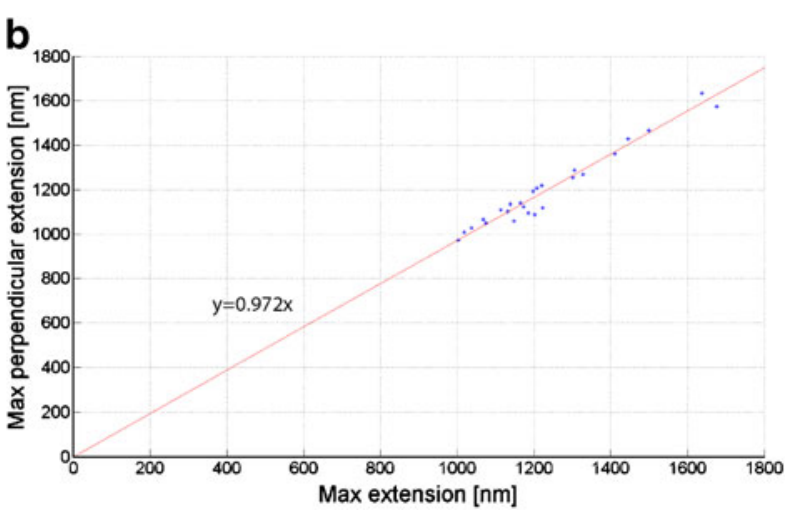

individual FISH signals, calculated as the ratio between the maximum-extension and the maximum perpendicular extension. The line of best fit has the equation $y=0.972 x$

\section{Discussion}

In a previous study, we have shown that localisation microscopy (SPDM) can be accomplished with conventional fluorochromes such as Alexa dyes or fluorescein derivatives (Reymann et al. 2008; Lemmer et al. 2009; Cremer et al. 2010). Besides their application in immunocytochemical staining methods, these dyes are widely used to specifically label chromatin structures by fluorescence in situ hybridisation (for review, see Cremer and Cremer 2001). Many of these structures, however, have a dimension too small for conventional light microscopical techniques. Examples for such structures in nuclear cell biology are small gene domains which may change their conformation due to differentiation or regulatory mechanisms. In this study, we have assessed and demonstrated the capability of the combination of fluorescence in situ hybridisation with localisation microscopy. Heterochromatin region Yq12 was labelled with a probe for DYZ2, a tandemly repeated sequence with a length of $2.47 \mathrm{~kb}$, which is distributed in about 2,000 copies over the entire length of band Yq12. In addition, we described a data processing procedure that minimises the loss of structural information present in the original data and hence produces an accurate representation of the measured structure. Several structural parameters were extracted, describing heterochromatin region Yq12 in far more detail than can be gained by conventional microscopical techniques. In particular, the extent of this region was 

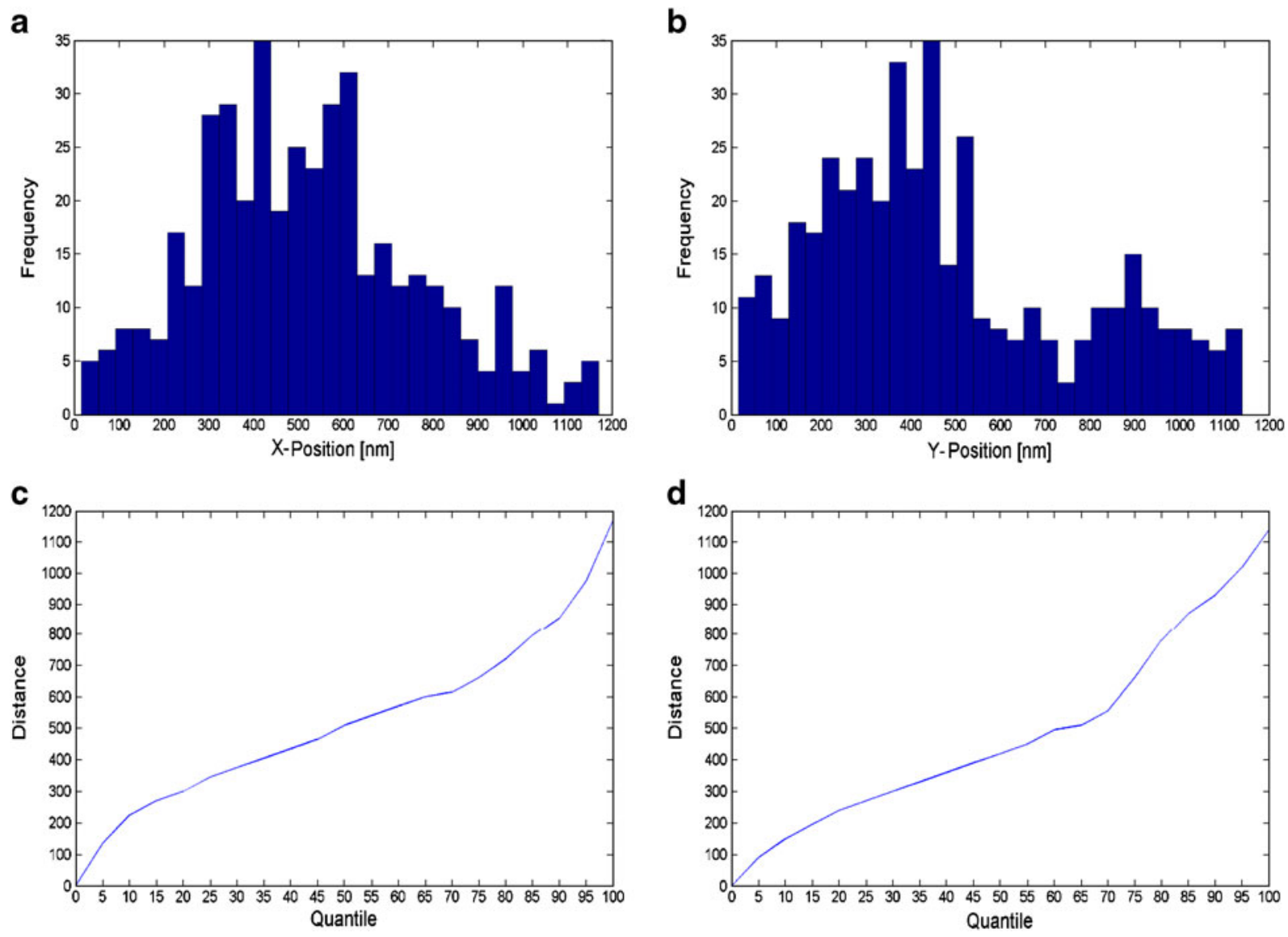

Fig. 11 Distribution of the $X$ and $Y$ positions of all single molecule signals obtained from the labelled region shown in Fig. 4. Figure $11(a, b)$ present the frequency distribution while

Fig. 11 (c, d) show the corresponding accumulated frequencies (quantiles). The distance is given in $\mathrm{nm}$

determined directly from the positions of individual hybridised molecules; the number of the molecules hybridised and detected was determined for each individual FISH-labelled region, despite a mean molecule distance much smaller than the conventional optical resolution $d_{\text {conv }}$ of approximately $200 \mathrm{~nm}$ : a density of 500 molecules $/ \mu m^{2}$ (Fig. 10a) corresponds to a mean distance of about $45 \mathrm{~nm}$, i.e. about five times smaller than $d_{\text {conv }}$. From the mean localisation accuracy $\sigma$ of about $20 \mathrm{~nm}$, an average optical resolution of about $2.35 \sigma$ corresponding to about $50 \mathrm{~nm}$ can be estimated. This means that so far the data do not allow drawing detailed conclusions on the substructure of the Yq12 domain. It is anticipated that this problem can be overcome by technical improvements of the SPDM microscopy technology presently in development

(Pertsinidis et al. 2010). Another current limitation is the number of detected molecules: From a FISH labelled Yq12 region containing a total number of 2000 repeats, only about 300-700 signals are obtained. Some reasons for this may be the labelling efficiency of the probes, the inherent optical sectioning capacity of the technique: a 'slice' of about $600 \mathrm{~nm}$ thickness was analysed while the entire Yq12 domain had a diameter of about $1 \mu \mathrm{m}$; or the efficiency of detection of bound probe molecules. Despite these limitations, the method allows currently a large variety of applications where the determination of relative molecule numbers is sufficent.

This paper presents the first detailed description of the application of localisation microscopy (SPDM) for the nanostructural analysis of specific FISH- 
a

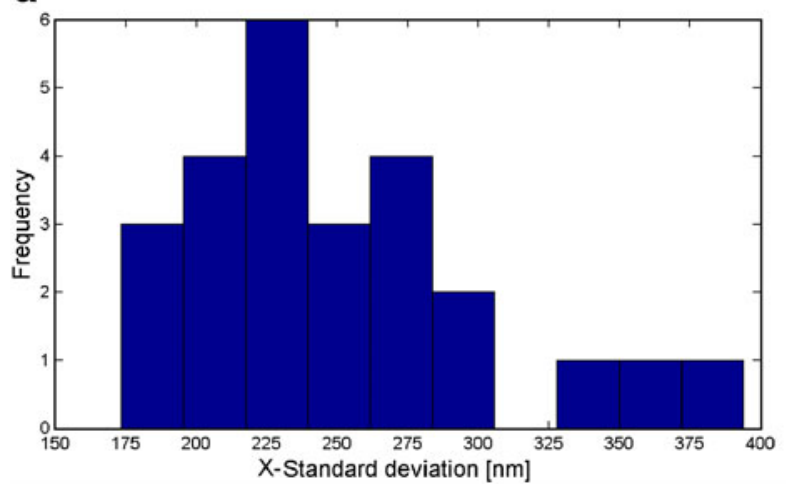

b

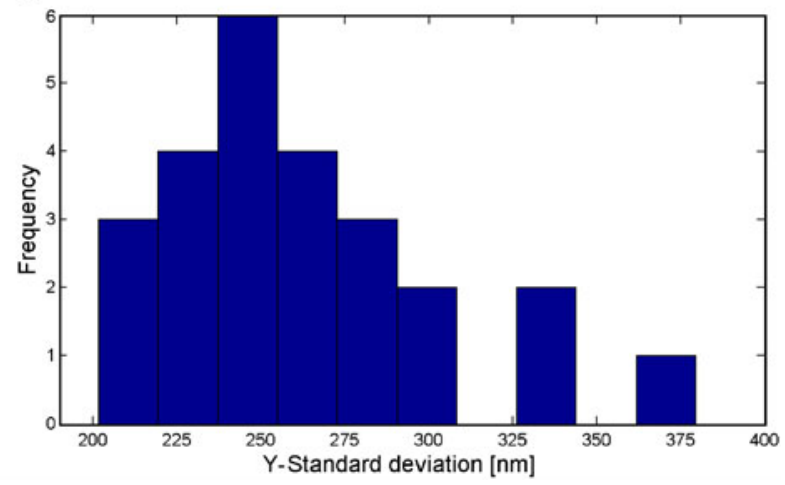

Fig. 12 Frequency histograms of the $X$ and $Y$ standard deviations of all molecules detected in the FISH labelled domains of all nuclei analysed $(n=25)$. Note that for a measure of the actual extent of the object in the individual directions, a factor of 2.35 is required

labelled chromatin domains. This approach may be combined with other SPDM techniques where fluorescent proteins were applied to study general features of nuclear nanostructure (Kaufmann et al. 2009; Gunkel et al. 2009; Bohn et al. 2010). For example, one could analyse specific domains of active/silenced genes or of gene deserts (Shopland et al. 2006) in the context of the histone distribution. Another interesting field of application is the analysis of break point regions induced e.g. by ionising radiation. In contrast to early attempts to use the SPDM methods (Esa et al. 2000,2001 ) where only a very few spectral signatures were available, the present FISH-SPDM technique described here would allow positioning multiple molecules in a nuclear area as small as $200 \mathrm{~nm}$ diameter. Another attractive field of application is to study condensation variabilities on the macromolecular resolution level, by artificially changing the degree of compaction of the chromatin. This can be done with chemicals such as Trichostatin A, an inhibitor of histone deactelylase, inducing decondensation of heterochromatin regions (Görisch et al. 2005), or Berenil, an AT-specific DNA ligand, inhibiting the condensation of the heterochromatin block on Yq12 (Haaf et al. 1989). The quantitative data obtained this way may be used to test numerical models of chromatin nanostructure (Odenheimer et al. 2009; Bohn et al. 2010) A further appealing perspective is to count copy numbers of specific DNA sequences on a single cell nucleus basis. So far, copy numbers are mainly assessed by PCR and array-based techniques, which have the drawback that they typically show an average over a large number of cells. Changes in copy numbers attract increasing interest, as it becomes more and more apparent that these contribute to several diseases, including cancer (Dear 2009).

The FISH localisation microscopy approach presented here is particularly suited for the analysis of small gene regions in intact cell nuclei. Many of these domains are thought to change their conformation due to activation but so far incontrovertible evidence is still elusive. Furthermore, extending the SPDM approach to two or more colours, each allowing the localization of a large number of molecules, will make possible to study on the nanoscale the interaction of specific chromatin structures such as the widely discussed problem of intermingling of chromosome territories (Branco and Pombo 2006; Rouquette et al. 2010).

The major concerns in the application of FISHbased SPDM to study nuclear nanostructure are changes induced by the fixation and denaturation process (Solovei et al. 2002b). This problem might be overcome in various ways, e.g., by using COMBO or PNA FISH with non-denaturating conditions (Hausmann et al. 2003; Schmitt et al. 2010) or by using thin cryosections (Branco and Pombo 2006).

Acknowledgements We gratefully acknowledge the support of the Deutsche Forschungsgemeinschaft (SPP1128) and of the European Union (In vivo molecular imaging Consortium, www.molimg.gr; 3D Genome project). Paul Lemmer is a fellow of the Hartmut Hoffmann-Berling International Graduate School of Molecular and Cellular Biology of the University Heidelberg and a member of the Excellence Cluster Cell 
Networks of the University of Heidelberg. We would like to thank our colleagues Patrick Müller, Rainer Kaufmann and Manuel Gunkel for stimulating discussions and great support.

\section{References}

Abbe E (1873) Beitraege zur Theorie des Mikroskops und der mikroskopischen Wahrnehmung. Arch Mikrosk Anat 9:411-468

Andresen M, Stiel A, Foelling J et al (2008) Photoswitchable fluorescent proteins enable monochromatic multilabel imaging and dual color fluorescence nanoscopy. Nat Biotechnol 26:1035-1040

Betzig E (1995) Proposed method for molecular optical imaging. Opt Lett 20:237-239

Betzig E, Patterson GH, Sougrat R et al (2006) Imaging intracellular fluorescent proteins at nanometer resolution. Science 313:1642-1645

Baddeley D, Carl C, Cremer C (2006) 4Pi microscopy deconvolution with a variable point-spread function. Appl Opt 45:7056-7064

Baddeley D, Batram C, Weiland Y et al (2007) Nanostructure analysis using spatially modulated illumination microscopy. Nat Protoc 2:2640-2646

Baddeley D, Jayasinghe ID, Cremer C et al (2009) Light-induced dark states of organic fluochromes enable $30 \mathrm{~nm}$ resolution imaging in standard media. Biophys J 96:L22-L24

Baddeley D, Weiland Y, Batram C et al (2010) Model based precision structural measurements on barely resolved objects. J Microsc 237:70-78

Bewersdorf J, Bennett BT, Knight KL (2006) H2AX chromatin structures and their response to DNA damage revealed by 4Pi microscopy. PNAS 103:18137-18142

Bohn M, Diesinger P, Kaufmann R et al (2010) Localization microscopy reveals expression-dependent parameters of chromatin nanostructure. Biophys J 99:1358-1367

Bornfleth H, Sätzler K, Eils R, Cremer C (1998) High-precision distance measurements and volume-conserving segmentation of objects near and below the resolution limit in threedimensional confocal fluorescence microscopy. J Microsc 189:118-136

Branco MR, Pombo A (2006) Intermingling of chromosome territories in interphase suggests role in translocations and transcription-dependent associations. PLoS Biol 4(5):e138

Bridger JM, Lichter P (1999) Analysis of mammalian interphase chromosomes by FISH and immunofluorescence. In: Bickmore WA (ed) Chromosome structural analysis. Oxford University, Oxford, pp 103-123

Burns DH, Callis JB, Christian GD et al (1985) Strategies for attaining super-resolution using spectroscopic data as constraints. Appl Opt 24:154-161

Cooke HJ (1976) Repeated sequences specific of human males. Nature 262:182-186

Cooke HJ, Schmidtke J, Gosden JR (1982) Characterisation of a human $\mathrm{Y}$ chromosome repeated sequence and related sequences in higher primates. Chromosoma 87:491-502

Cremer C, Cremer T (1978) Considerations on a laserscanning-microscope with high resolution and depth of field. Microsc Acta 81:31-44
Cremer T, Cremer C (2001) Chromosome territories, nuclear architecture and gene regulation in mammalian cells. Nat Rev Genet 2:292-301

Cremer C, Hausmann M, Bradl J, Rinke B: German Patent Application No. 196.54.824.1/DE, submitted Dec 23, 1996, European Patent EP 1997953660, 08.04.1999, JapanesePatent JP 1998528237, 23.06.1999, United States Patent US09331644, 25.08.1999.

Cremer C, Edelmann P, Bornfleth H (1999) Principles of spectral precision distance confocal microscopy of molecular nuclear structure. In: Jähne B, Haußecker H, Geißler P (eds) Handbook of Computer Vision and Applications, 3rd edn. Academic, New York, pp 839-857

Cremer C, Failla AV, Albrecht B (2001) Far field light microscopical method, system and computer program product for analyzing at least one object having a subwavelength size. US Patent 7,298,461 B2

Cremer C, von Ketteler A, Lemmer P et al (2010) Far field fluorescence microscopy of cellular structures@ molecular resolution. In: Diaspro A (ed) Nanoscopy and Multidimensional Optical Fluorescence Microscopy. Taylor and Francis, New York, pp3/1-3/35

Dear PH (2009) Copy-number variation: the end of the human genome? Trends Biotechnol 27:448-454

Edelmann P, Cremer C (2000) Improvement of confocal spectral precision distance microscopy (SPDM). Proc SPIE 3921:313-320

Edelmann P, Esa A, Hausmann M, Cremer C (1999) Confocal laser-scanning fluorescence microscopy: In situ determination of the confocal point-spread function and the chromatic shifts in intact cell nuclei. Optik 110:194-198

Egner A, Jakobs S, Hell SW (2002) Fast 100-nm resolution three-dimensional microscope reveals structural plasticity of mitochondria in live yeast. PNAS 99:3370-3375

Egner A, Geisler C, von Middendorff C et al (2007) Fluorescence nanoscopy in whole cells by asynchronous localization of photoswitching emitters. Biophys J 93:3285-3290

Esa A, Edelmann P, Trakhtenbrot L et al (2000) Threedimensional spectral precision distance microscopy of chromatin nanostructures after triple-colour DNA labelling: a study of the BCR region on chromosome 22 and the Philadelphia chromosome. J Microsc 199:96-105

Esa A, Coleman AE, Edelmann P et al (2001) Conformational differences in the 3D-nanostructure of the immunoglobulin heavychain locus, a hotspot of chromosomal translocations in B lymphocytes. Cancer Genet Cytogenet 127:168-173

Fölling J, Bossi M, Bock $\mathrm{H}$ et al (2008) Fluorescence nanoscopy by ground-state depletion and single-molecule return. Nat Methods 5:943-945

Geisler C, Schönle A, von Middendorff C et al (2007) Resolution of $\lambda / 10$ in fluorescence microscopy using fast single molecule photo-switching. Appl Phys A 88:223-226

Gerdes J, Schwab U, Lemke H et al (1983) Production of a mouse monoclonal antibody reactive with a human nuclear antigen associated with cell proliferation. Int $\mathrm{J}$ Cancer 31:13-20

Görisch SM, Wachsmuth M, Fejes Tóth K et al (2005) Histone acetylation increases chromatin accessibility. J Cell Sci 118:5825-5834 
Gunkel M, Erdel F, Rippe K et al (2009) Dual color localization microscopy of cellular nanostructures. Biotechnol J 4:927938

Gustafsson MG (2000) Surpassing the lateral resolution limit by a factor of two using structured illumination microscopy. J Microsc 198:82-7

Haaf T, Feichtinger W, Guttenbach M et al (1989) Berenilinduced undercondensation in human heterochromatin. Cytogenet Cell Genet 50:27-33

Hänninen PE, Hell SW, Salo J et al (1995) Two-photon excitation 4Pi confocal microscope: Enhanced axial resolution microscope for biological research. Appl Phys Lett 66:1698-1700

Hausmann M, Winkler R, Hildenbrand G et al (2003) COMBO-FISH: specific labeling of nondenatured chromatin targets by computer-selected DNA oligonucleotide probe combinations. Biotechniques 35:564-577

Hell SW (2003) Towards fluorescence nanoscopy. Nat Biotechnol 2:1347-1355

Hell SW (2007) Far-field optical nanoscopy. Science 316:11531158

Hell SW, Wichmann J (1994) Breaking the diffraction resolution limit by stimulated emission: stimulatedemission-depletion fluorescence microscopy. Opt Lett 19:780-782

Hell SW, Lindek S, Cremer C et al (1994) Measurement of the 4Pi-confocal point spread function proves $75 \mathrm{~nm}$ axial resolution. Appl Phys Lett 64:1335-1337

Heilemann M, Herten DP, Heintzmann R et al (2002) Highresolution colocalization of single dye molecules by fluorescence lifetime imaging microscopy. Anal Chem 74:3511-3517

Heintzmann R, Cremer C (1999) Laterally modulated excitation microscopy: improvement of resolution by using a diffraction grating. Proc SPIE 3658:185-195

Hendrix J, Flors C, Dedecker P et al (2008) Dark states in monomeric red fluorescent proteins studied by fluorescence correlation and single molecule spectroscopy. Biophys J 94:4103-4113

Hess ST, Girirajan TPK, Mason MD (2006) Ultra-High resolution imaging by fluorescence photoactivation localization microscopy. Biophys J 91:4258-4272

Högbom JA (1974) Aperture Synthesis with a Non-Regular Distribution of Interferometer Baselines. AandAS 15:417426

Kaufmann R, Lemmer P, Gunkel M et al (2009) SPDM-Single molecule super-resolution of cellular nanostructures. Proc SPIE 7185:7185-J

Lacoste TD, Michalet X, Pinaud F et al (2000) Ultrahighresolution multicolor colocalization of single fluorescent probes. PNAS 97:9461-9466

Lamond AI, Earnshaw WC (1998) Structure and function in the nucleus. Science 280:547-553

Lang M, Jegou T, Chung I et al (2010) Three-dimensional organization of promyelocytic leukemia nuclear bodies. J Cell Sci 123:392-400

Lemmer P, Gunkel M, Baddeley D et al (2008) SPDM: light microscopy with single-molecule resolution at the nanoscale. Appl Phys B 93:1-12

Lemmer P, Gunkel M, Weiland Y et al (2009) Using conventional fluorescent markers for far-field fluorescence localization nanoscopy allows resolution in the $10-\mathrm{nm}$ range. J Microsc 235:163-171

Matheron G (1975) Random sets and integral geometry. Wiley, New York

Misteli T (2005) Concepts in nuclear architecture. Bioessays $27: 477-87$

Misteli T (2007) Beyond the sequence: cellular organization of genome function. Cell 128:787-800

O'Brien TP, Bult CJ, Cremer C et al (2003) Genome function and nuclear architecture: From gene expression to nanoscience. Genome Res 13:1029-1041

Odenheimer J, Heermann DW, Kreth G (2009) Brownian dynamics simulations reveal regulatory properties of higher-order chromatin structures. Eur Biophys J 38:749-756

Pertsinidis A, Zhang Y, Chu S (2010) Subnanometre singlemolecule localization, registration and distance measurements. Nature 466:647-651

Patterson GH, Lippincott-Schwartz J (2002) A photoactivatable GFP for selective photolabeling of proteins and cells. Science 297:1873-1877

Rauch J, Hausmann M, Solovei I et al (2000) Measurement of local chromatin compaction by Spectral Precision Distance microscopy. Proc SPIE 4164:1-9

Rayleigh L (1896) On the theory of optical images, with special reference to the microscope. Philos Mag 42:167-195

Reymann J, Baddeley D, Gunkel M et al (2008) High-precision structural analysis of subnuclear complexes in fixed and live cells via spatially modulated illumination (SMI) microscopy. Chromosome Res 16:367-382

Rouquette J, Cremer C, Cremer T, Fakan S (2010) Functional nuclear architecture studied by microscopy: present and future. Int Rev Cell Mol Biol 282:1-90

Rust MJ, Bates M, Zhuang X (2006) Sub-diffraction-limit imaging by stochastic optical reconstruction microscopy (STORM). Nat Methods 3:793-796

Schermelleh L, Carlton PM, Haase S et al (2008) Subdiffraction multicolor imaging of the nuclear periphery with $3 \mathrm{~d}$ structured illumination microscopy. Science 320:1332-1336

Schmid M, Guttenbach M, Nanda I et al (1990) Organization of DYZ2 repetitive DNA on the human $\mathrm{Y}$ chromosome. Genomics 6:212-218

Schmidt M, Nagorni M, Hell SW (2000) Subresolution axial distance measurements in far-field fluorescence microscopy with precision of 1 nanometer. Rev Sci Instrum 71:2742-272745

Schmidt R, Wurm CA, Jakobs S et al (2008) Spherical nanosized focal spot unravels the interior of cells. Nat Methods 5:539-544

Schmitt E, Schwarz-Finsterle J, Stein S et al (2010) Combinatorial Oligo FISH: Directed Labeling of Specific Genome Domains in Differentially Fixed Cell Material and Live Cells. Methods Mol Biol 659:185-202

Schrader M, Meinecke F, Bahlmann K et al (1995) Monitoring the excited state of a dye in a microscope by stimulated emission. Bioimaging 3:147-153

Serra J (1982) Image analysis and mathematical morphology. Academic, London

Shopland LS, Lynch CR, Peterson KA et al (2006) Folding and organization of a contiguous chromosome region according to the gene distribution pattern in primary genomic sequence. J Cell Biol 174:27-38 
Sinnecker D, Voigt P, Hellwig N et al (2005) Reversible photobleaching of enhanced green fluorescent proteins. Biochemistry 44:7085-7094

Solovei I, Walter J, Cremer M et al (2002a) FISH on threedimensionally preserved nuclei. In: Beatty B, Mai S, Squire J (eds) FISH. Oxford University, Oxford, pp 119-157

Solovei I, Cavallo A, Schermelleh L et al (2002b) Spatial preservation of nuclear chromatin architecture during three-dimensional fluorescence in situ hybridization (3DFISH). Exp Cell Res 276:10-23

Thompson RE, Larson DR, Webb WW (2002) Precise nanometer localization analysis for individual fluorescent probes. Biophys J 82:2775-2783

Van Oijen AM, Köhler J, Schmidt J, Brakenhoff GJ (1998) 3-dimensional super-resolution by spectrally selective imaging. Chem Phys Lett 292:183-187 